\title{
Kirchliche Kommunikationskultur
}

\section{Überlegungen zur Kirche in der Welt der Massenmedien}

\author{
von Johann Baptist Metz
}

\section{Konzilsperspektiven}

Vielleicht war das jüngste Konzil der Kirche doch erst ein Anfang, ein bislang unabgegoltener Anfang. Vielleicht. Für mich gilt das jedenfalls hinsichtlich zweier zentraler Anstöße dieses Konzils, die es nicht etwa ängstlich zu blockieren, sondern in wagender, in offensiver Treue zu dieser konziliaren Kirchenvision auszugestalten gilt. Der eine zielt auf den Innenraum der Kirche. Er läßt sich als Entdeckung oder Bekräftigung der unhintergehbaren Autorität der Glaubenden in der Kirche kennzeichnen. Während das Kirchenbild des I. Vatikanums noch deutlich vom Verhältnis zwischen Souverän und Untertan geprägt ist, steht beim Kirchenverständnis des jüngsten Konzils das biblische Bild vom wandernden Gottesvolk im Vordergrund. Und seit diesem Konzil probt nun dieses Gottesvolk - mit guten biblischen Gründen - den aufrechten Gang. Der ist freilich schwer zu lernen und kaum ohne Stürze, ja ohne Abstürze ins Weglose und Abwegige zu haben. Aber wie man einem Kind doch nicht zureden darf, das Laufen erst gar nicht zu lernen, weil es dabei nach allem Ermessen ohne Stürze nicht abgeht, darf die Kirche nicht auf den aufrechten Gang der Glaubenden verzichten wollen, nur um des Wagnisses willen, das in ihm steckt.

Diese profane Metapher vom aufrechten Gang will kirchlich einen entscheidenden Umstand zum Ausdruck bringen: daß nämlich nur aufrecht Gehende auch freiwillig Kniende und mit Frohsinn Dankende sein können; und daß kritische Einsprüche und Rückfragen keineswegs von sich aus Anzeichen kirchlicher Illoyalität sind. Wie heißt es doch in einem der Konzilsdokumente („Über die Religionsfreiheit“, Nr. 3): „Die Wahrheit muß auf eine Weise gesucht werden, die der Würde der menschlichen Person und ihrer Sozialnatur eigen ist", will sagen: Es geht nun nicht (mehr) um die Unterordnung der Glaubenden unter einen abstrakten, einen subjektlosen Wahrheitsbegriff. Das Recht der Wahrheit, auf das die Kirche sich allemal beruft, ist zunächst das Recht der Person in ihrer Wahrheit. Diese urchristliche Einsicht ist nicht primär durch die Kirche, eher schon ohne sie, in gewisser Hinsicht nur gegen sie gerettet worden. Offensichtlich kehrt das Evangelium auch über die fremde Prophetie der sog. profanen Welt in die Kirche ein. Wo die Kirche ihre Augen nur nach innen richtet, verliert sie sich selbst aus dem Blick.

Der zweite Anstoß des Konzils zielt auf die weltweite Sendung der Kirche. Im jüngsten Konzil zeichnet sich nämlich keimhaft eine neue Phase der Kirchengeschichte $\mathrm{ab}$. Sie läßt sich - erneut äußerst gerafft - so kennzeichnen:

DDr. J. B. Metz ist Ordinarius für Fundamentaltheologie an der Westfälischen-WilhelmsUniversität in Münster. 
Nach einer zeitlich relativ kurzen, aber für die theologisch-historische Identität der Kirche fundamentalen Epoche des Judenchristentums war die Kirche über nahezu zweitausend Jahre einem relativ einheitlichen Kulturraum verpflichtet, nämlich dem abendländisch-europäischen. Sie weitet sich heute zu einer kulturell vielfach verwurzelten Weltkirche. In diesem Sinne steht sie in der vielleicht tiefgreifendsten Zäsur seit urchristlicher Zeit. Sie ist auf dem Weg von einer kulturell mehr oder weniger einheitlichen, also kulturell monozentrischen europäischen Kirche zu einer kulturell vielfach verwurzelten und in diesem Sinn kulturell polyzentrischen Weltkirche. Kirche will nun nicht mehr nur eine europäische „Kirche für andere" sein, sondern dezidiert auch eine „Kirche der anderen". Dieser Übergang zeichnet sich - als Anfang eines Anfangs - im jüngsten Konzil ab.

In diesem Zusammenhang möchte ich Sie auf zwei Fragen aufmerksam machen, die uns später erneut beschäftigen werden. Man könnte nämlich erstens - den Eindruck haben, daß die Kirche in einer für sie typischen Ungleichzeitigkeit die Würde fremder Kulturen und Lebenswelten gerade dann entdeckt, wenn diese Kulturen selbst schon in ihrer Substanz bedroht sind. In welchem Sinn gibt es heute überhaupt noch einen realen kulturellen Polyzentrismus in der Welt? Wird er nicht immer mehr aufgelöst oder allenfalls folklorisiert von jener profanen Europäisierung, von jener westlichen Rationalität, ihrer Technik und ihrer Informations- und Kommunikationsindustrie, die heute unsere ganze Welt umspannt und die nicht nur die Praxis, sondern offensichtlich auch die Mentalitäten der Völker verändert? Ein Balinese, der ein Auto fährt, ist schon ein halber Europäer. Und ein Indio, der sich keine Geschichten mehr erzählen läßt, sondern fernsieht, auch. Entdecken wir womöglich die Kirche der anderen in einer Zeit, in der es die andern in ihrer Andersheit kaum mehr gibt? Oder in der sie, verstört durch die europäische Technik- und Kulturinvasion, quasi fundamentalistisch ihrerseits jede Kommunikation abbrechen?

Die zweite Frage gilt der empirischen Grundsituation der Kirche in der heutigen Welt. Sie zeigt, daß die Kirche in einem höchst komplexen, teilweise geradezu gegenläufigen Prozeß steht: sie ist erstmals auf dem Weg, reale weltumspannende Kirche zu werden; im gleichen Zug aber gerät sie überall auf der Welt und nicht zuletzt bei uns in Europa immer mehr in eine Minderheitensituation, in eine globale Diaspora. Diese Diasporasituation nimmt zu, nicht ab. Hatten wir denn gedacht, die Kirche finde, wie einst das frühafrikanische Christentum in Europa, nun nach Europa eine flächendeckende Zukunft in Asien, in Afrika oder zumindest in Lateinamerika? Wir wissen heute, daß dem nicht so ist, nicht so sein wird. Eine Evangelisierung der Welt, die sich solchen Vorstellungen verschriebe, müßte scheitern. Und im Blick auf Europa? Beunruhigt von der dramatisch wachsenden Diasporasituation der Kirche in Europa ist gegenwärtig viel die Rede von einer "Neuevangelisierung Europas". Aber wie? Will man womöglich die christliche Diaspora in Europa in ein vormodernes, vorreformatorisches „christliches Abendland“ zurückevangelisieren?

Gewiß ist das Christentum sozusagen strukturell eingebaut in die Geschichte und den Geist Europas. Aber wie entziehen wir uns dem Eindruck, das Christentum gehöre zwar zu den Voraussetzungen, gewissermaßen zu den hi- 
storischen und kulturellen Hintergrundannahmen des europäischen Geistes, nicht aber zu seinen gegenwärtigen Lebensinhalten und Handlungsimperativen? Wie entwinden wir das Christentum in Europa seiner puren Historisierung? Oder auch und vor allem seiner reinen Ästhetisierung? Ich nenne diese Fragen, weil ich die Schwierigkeiten benennen und mit Ihnen teilen möchte, mit denen heute jede substantielle kirchliche Kommunikation zu rechnen hat.

\section{Kommunikation in der Einen Welt}

Die Erfahrung der Einen Welt begegnet der Kirche nicht von außen. Sie ist selbst, wie gesagt, erstmals auf dem Weg zur realen Weltkirche. Ob sie als kulturell polyzentrische Weltkirche gelingt, hängt nicht zuletzt daran, ob sie in sich selbst eine anspruchsvolle kommunikative Kultur entfaltet: eine Kultur der Anerkennung der andern in ihrem Anderssein. Es geht dabei nicht um eine Verklärung der andern, aber doch um den Respekt vor ihren unentschlüsselten Geheimnissen. Der universale Sendungsauftrag der Kirche ist, in einem Wort, Weltentdeckung mit den Augen Jesu. Jesus, aber lehrte eine Nächstenliebe, in der die Nächsten nicht einfach die Nahen sind, sondern - vor allem - die anderen, die unbegriffenen fremden anderen. Er wollte uns mit „Augen für die anderen“ ausstatten. „Aufwachen, die Augen öffnen“: diese Aufforderung durchzieht alle biblischen Aussagen. Sie könnte geradezu als kategorischer Imperativ des Christentums gelten. Entgegen der landläufigen Rede vom „blinden Glauben" bestehen die biblischen Traditionen, besteht vor allem Jesus selbst auf Sichtbarkeit und auf gesteigerter Wahrnehmungs- und Anerkennungsbereitschaft. Darin wurzelt die gesuchte kommunikative Kultur.

Lassen Sie mich die Frage nach dieser kommunikativen Kultur heute wegen des bevorstehenden 500jährigen Jubiläums der sog. Entdeckung Amerikas - im Blick auf das Verhältnis Europa - Lateinamerika erörtern. Mit welchen Augen wurde dieser Kontinent „entdeckt"? Spielte dabei die urchristliche kommunikative Kultur der Anerkennung der anderen in ihrem Anderssein die maßgebende Rolle? Oder war diese „Entdeckung" nicht weit mehr und allzu sehr von einer fragwürdigen Angleichungs- und Beherrschungskultur geleitet, die kaum Augen hatte für die Spur Gottes in der Andersheit der anderen und die deshalb diese unbegriffenen andern immer wieder zu Opfern machte? $\mathrm{Zu}$ Beginn der Zeit, die wir in Europa die "neue" nennen und in der auch die großen europäischen "Entdeckungen" anheben, vorweg die des Kolumbus, entfaltete sich - keimhaft und von vielen religiösen und kulturen Symbolen überlagert eine Art Herrschaftsanthropologie: Der Mensch verstand sich immer mehr als herrschaftliches, als unterwerfendes Subjekt gegenüber der Natur. An dieser herrscherlichen Unterwerfung, an dieser Machtergreifung über die Natur bildete sich seine Identität. Seine Augen blickten nach unten. Seine Logik wurde eine Logik der Beherrschung, nicht der Anerkennung, eine Logik allenfalls der Assimilation, nicht aber der Alterität.

Ist dieses Zeitalter der europäischen Herrschaftsanthropologie endgültig vorüber? Inzwischen sind ihre Opfer annähernd bekannt, wenn auch nur spärlich erinnert. Ist das europäische Zeitalter, in dem die Neugierde durchdrungen ist vom Willen zur Macht, vorbei? Ist das Zeitalter der europäischen Unempfind- 
lichkeit und Unirritierbarkeit endlich vorüber? Kommt ein anderes Europa zur Geltung? Schließlich trägt der Geist der europäischen Moderne noch ganz andere als diese herrschaftlichen Züge. So bildete sich in ihm gleichzeitig eine Anthropologie heraus, die in einem ganz anderen Sinn praktisch sein will, nämlich als Freiheit, auch als Freiheit der jeweils anderen und damit als Gerechtigkeit: eine Anthropologie der universalen Menschenrechte, die auf die subjekthafte Freiheit und Würde aller pocht. Ist dieser nichtherrscherliche Universalismus die Signatur des europäischen Geistes für 1992? Und des kirchlichen Geistes für 1992? Und welche Rolle spielen dabei die modernen Massenmedien, die ja in ihrer Wurzel selbst ein europäisches Projekt sind?

Ich will hier einmal unterstellen, daß die audiovisuellen Medien wirklich sehen lehren und nicht nur zuschauen. In diesem Falle hätten sie für die angeschnittene Frage eine besondere Bedeutung. "Sieh hin und du weißt" hat der Philosoph Hans Jonas formuliert und dabei das Sehen, die Augen für die anderen zur Wurzel für eine neue Anerkennungsbereitschaft wie für eine neue Art universalistischer Moral gemacht.

"Sieh hin und du weißt": du weißt um die Würde der fremden anderen, weißt, daß z. B. die Indios Lateinamerikas sich nicht nur deswegen so schwer tun mit unserer westlichen Zivilisation, weil sie, wie wir gern sagen oder doch vermuten, einfach noch „unterentwickelt“ sind, sondern zunächst einmal, weil sie anders sind und weil dieses Anderssein seine zu respektierenden Geheimnisse hat. „Sieh hin und du weißt": du weißt, daß die Trauerantlitze dieser Indios auch von einer eigentümlichen Stärke geprägt sind, von einer geheimen Resistenz - etwa gegen die rasende Beschleunigung der Zeit, die wir 'Europäer inszeniert haben und in der wir immer mehr uns selbst abhanden kommen; gegen jenes Vergessen bzw. jenes Vergessen des Vergessens, das bei uns den Namen „Fortschritt“ und „Entwicklung“ trägt ...

"Sieh hin und du weißt": dieser Satz hat auch eine moralische und politische Pointe. Was wir "Gewissen“ nennen, ist ein Wissen, das aus solchem Hinsehen stammt. Aus diesem Hinsehen und diesem Angeblicktwerden, in dem wir über unsere eingeborenen Narzißmen hinauswachsen, wächst uns ein Verantwortungsgefühl zu für Zustände und Situationen, die wir nicht unbedingt selbst verursacht haben. Die fremden Augen klagen eine Solidarität ein, die weit über unsere Familien- und Nachbarschaftsmoral hinausgeht. Wer hätte das noch nicht erfahren, wenn er angeblickt wurde von den tränenlosen Augen, dem traumlosen Unglück der Kinder und Frauen aus der Dritten Welt?

Und das soll nicht von elementarer Bedeutung sein für die Kommunikationskultur einer Kirche, die heute ja immer weniger eine Dritte-Welt-Kirche "hat" und immer mehr eine solche "ist" - ?

Indes, wenn man für eine solche Kommunikationskultur die Massenmedien beansprucht, tauchen auch Bedenken auf und Rückfragen. Zunächst im Blick auf das Medium selbst. Privilegiert, befördert z. B. das Fernsehen in seiner Langzeitwirkung nicht doch zu sehr das zerstreute, das vergleichgültigende $\mathrm{Zu}$ schauen? Verbreitet und unterstützt es wirklich anerkennungsbereite, verant- 
wortungsbewußte Mündigkeit oder produziert es - auf Dauer - nicht eine neue, gewissermaßen eine sekundäre Unmündigkeit, die gespeist ist von dem Eindruck, daß wir heute zwar mehr als je über alles informiert sind, vor allem auch über das, was uns bedroht und über alle Krisen und Schrecken in der Welt, daß uns aber der Schritt vom Wissen zum Tun, von der Information zum Handeln noch nie so aussichtslos erschien wie heute. Eine Art Krisengewöhnungsdenken breitet sich aus. Wir gewöhnen uns an die Armutskrisen in der Welt, die sich immer mehr zu verstetigen scheinen und die wir deshalb achselzuckend an eine anonyme, an eine subjektlose gesellschaftliche Evolution delegieren. Hängt das aber, so frage ich mich, nicht auch an der Art, wie wir über das Medium "sehen" und "wahrnehmen": an diesem quasi körperlosen Sehen im Fernsehsessel, das die volle leibhaftige Präsenz, das Dabeisein mit allen Sinnen überflüssig oder jedenfalls sekundär erscheinen läßt? Ist vielleicht die Suspension dieser leibhaftigen Präsenz nicht doch auch eine Suspension in der Ebene der Kommunikation und der Solidarität? Bestärkt uns am Ende das audiovisuelle Medium in der Illusion, wir könnten anteilnehmen und teilen, ohne bei uns selbst, in unserem leibhaftigen Leben, in unserem sinnenhaft-sozialen Dasein etwas zu bewegen und zu ändern?

Natürlich tauchen auch Fragen und Sorgen auf, wenn es um die Auswirkungen der Massenmedien auf die Dritte Welt geht, nicht zuletzt wiederum auf Lateinamerika. Die großen westlichen Vier - AP und UPI in den USA, Reuter in England und AFP in Frankreich - bestreiten bekanntlich zu über $70 \%$ das Informations- bzw. Nachrichtenangebot in der Welt. Das lateinamerikanische Fernsehen ist bis heute fest in nordamerikanischer Hand; nur Mexiko und vor allem Brasilien verkaufen ihrerseits Programme auf regionalen Märkten. Kurzum: es gibt die Gefahr einer zweiten, einer sekundären Kolonisation, einer Kolonisation, die viel schneller und wirksamer die Seelen der Menschen dieser Länder erreicht als es die militärische und lógistische Übermacht der früheren Kolonialtruppen je vermochte. Erst letzte Woche sagte mir Gustavo Gutiérrez aus Lima: durch diese westlich geprägten Massenmedien komme es zu einer massiven Entwurzelung, zu einem Umsturz der Werte in der. Welt der Armen seines Landes - und noch weit mehr als bei uns zu einem sog. Realitätswahrnehmungsverlust. Die Modernisierung der Köpfe durch die moderne Massenmedienkultur produziert ein imaginäres Bewußtsein, und das wirkt eben an der untersten Grenze des Lebens - in den Elendsvierteln, in den Favelas - wie der einzige noch verfügbare Traum und Trost. „Das Fernsehen bei uns", sagte eine brasilianische Medienexpertin, „ist ein großer Supermarkt, der an die Armen imaginäre Güter verteilt.“

Ich weiß, es gibt inzwischen aus den Ländern der Dritten Welt - analog zum Ruf nach einer neuen Weltwirtschaftsordnung - auch das immer dringlicher werdende Postulat einer "neuen internationalen Informations- und Kommunikationsordnung", um die Gefahr des Medienkolonialismus zu verringern. Wenn man diese Forderung energisch unterstützt - was der Kirche sehr wohl anstünde - wird man freilich auch darauf achten müssen, daß es eine Gefährdung ethnischer und kultureller Identitäten in diesen armen Ländern nicht nur von seiten der Ersten Welt und ihrer Medienübermacht gibt, sondern auch innerhalb der Länder der Dritten Welt selbst, dort vor allem, wo eine rigorose "nationalstaatli- 
che Hermeneutik" im Medienbereich zur Geltung kommt, die viele ethnischkulturelle Minderheiten im Namen der nationalen Integration zum Verstummen und schließlich zum Verschwinden bringt usw. Hier gibt es offensichtlich Fragen und Probleme, auf die auch Kompetentere als ich zumeist nur spärliche Antworten haben. Vielleicht müßte in vielen der angesprochenen Länder vor allem der Rundfunk unterstützt werden, das Radio, wegen seiner größeren Nähe zur sog. oralen Kultur dieser Völker; und vor allem die Printmedien, in erster Linie die Alphabetisierungsliteratur, damit diese Menschen nicht durch die Massenmedien um ihre eigene Sprache gebracht werden, ehe sie sich selbst erstmals zur Sprache gebracht, erstmals sich selbst alphabetisiert haben. Es gibt eben nicht nur Chancen, sondern vor allem auch Tragödien der Ungleichzeitigkeit in einer massenmedial immer rapider zusammenwachsenden Welt.

Und bei uns hierzulande, hier in Europa? Wie die moderne Wissenschaft, wie die Technik ist die massenmediale Kommunikation eigentlich ein europäisches Projekt. Feiert so der alte unsensible Eurozentrismus in den Massenmedien seine letzten Triumphe? Doch das sozial und kulturell Gefährliche am Eurozentrismus wurzelt, genau besehen, nicht darin, daß wir zu viel Europa „exportieren“, sondern zu wenig, genauerhin nur das halbierte Europa, das Europa einer rationalen und kommerziellen Herrschaftskultur und nicht das Europa jener Kommunikationskultur, die in der Anerkennung der Freiheit und Würde der anderen praktisch werden will und die deshalb diese anderen nicht nur zu ihrem Ausdruck, sondern auch immer mehr zu ihrem Recht bringen muß. An der Überwindung dieser Halbierung mitzuarbeiten, wäre auch die zentrale Aufgabe einer kirchlichen Kommunikationskultur, die den Herausforderungen einer sozial geteilten und kulturell polyzentrischen Weltkirche gewachsen sein will.

\section{Kommunikation in der globalen Diaspora}

Kirche, Christentum in globaler Diaspora, nicht zuletzt und immer mehr bei uns in Europa: auch darin liegt eine Herausforderung an die kirchliche Kommunikationskultur. Die mehr gefühlte als offen diskutierte Minderheitensituation steigert die Gefahr des Ghettodenkens und der Sektenmentalität in der Kirche. Doch nicht eigentlich die Minorität, sondern die Mentalität definiert die Sekte und das Ghetto in einem theologischen Sinn. Minorität braucht die Kirche letztlich weder zu fürchten noch sich ihrer zu schämen, es sei denn, sie hielte sich selbst für die innerweltliche Vollstreckerin der von ihr bezeugten universalen Heilsgeschichte und mißverstehe sich so als eine ihre eigene Hoffnung ersetzende religiöse Ideologie, in der die bleibende endzeitliche Differenz zwischen der Kirche und dem Reich Gottes unterschlagen ist.

Mehr als bisher muß eine kirchliche Kommunikationskultur heute ins Auge fassen, daß die flächendeckenden volkskirchlichen Strukturen in Europa immer mehr verschwinden, daß es zum Überleben des lebendigen Christentums immer dringlicher neuer kirchlicher Lebensformen bedarf und einer nüchternen, unsentimentalen Diasporapastoral, die sich in die Stein- und Seelenwüsten unserer Großstädte und an die verkabelten Arbeitsplätze unserer High-Tec-Welten wagt, um auch dort den messianischen Tugenden auf der Spur zu sein, jenen 
Gegen-Tugenden zum Markt- und Verwertungsdenken, für die man buchstäblich nichts bekommt, die aber nur der heutzutage für verschwunden oder für verödet hält, der sein Christentum ohnehin als einen Kult der Verzweiflung am modernen Menschen pflegt. Audiovisuelle Berichte aus der Diaspora unangepaßter Empfindungen und Einstellungen: das scheint mir eine der wichtigsten künftigen Aufgaben einer kirchlichen Kommunikationskultur in Europa zu sein.

Widerstehen muß eine kirchliche Kommunikationskultur den Symptomen einer neuen Sekten- und Ghettomentalität in der Öffentlichkeit der Kirche selbst:

- dem Trend etwa zu einem kirchlichen Fundamentalismus, der jeden Widersprechenden und Andersdenkenden von vornherein entwürdigt,

- den Anzeichen eines puren Traditionalismus, der alles Gefährliche und Befreiende am christlichen Gedächtnis den Selbsterhaltungsinteressen der kirchlichen Institutionen unterordnet;

- der wachsenden Unfähigkeit bzw. Unwilligkeit, neue Erfahrungen zu machen und sie in schmerzlich-kritischer Assimilation in das Selbstverständnis der Kirche einzubauen;

- einer zelotisch angeschärften Sprache und einer verständigungsunfähigen Militanz bzw. einer allzu autoritären Selbstsicherheit bei innerkirchlichen Auseinandersetzungen;

- der Verwechslung von Kirchlichkeit mit einem freud- und humorlosen Zelotentum;

- der Ausbreitung eines Loyalitätsüberdrucks bzw. der Anzeichen von Überängstigung im kirchlichen Leben;

- dem Drang schließlich, sich nur unter Gleichgesinnten aufzuhalten, der Gefahr einer künstlichen Isolation der Verkündigungssprache, die zur reinen kommunikationsunfähigen Binnensprache wird mit einer typischen Sektensemantik.

Solchen und ähnlichen Symptomen haben sich die Träger einer kommunikativen Kultur in der Kirche entgegenzustellen. Zu ihnen gehören - neben den Theologen - auch die Medienjournalisten in der Kirche. Ihr kritischer Einspruch legitimiert sich einmal aus der erwähnten Autorität aller Glaubenden, besonders aber auch aus dem Umstand, daß gar nicht sie die primären Subjekte dieser Kritik sind und sein sollen, sondern die Mehrzahl des in stimmlosen Stimmungen verharrenden und zuweilen in sprachloser Observanz gehaltenen Kirchenvolkes. Gewiß gibt es unter Theologen und Journalisten immer auch solche, die zur Selbstinthronisation neigen und die deshalb um ihrer selbst willen in die Medien drängen, um dort ihre Sondermeinungen zu verkünden und anstelle von professionell erarbeiteten Informationen eine längst zum Klischee erstartte Kirchenkritik vorzutragen. Doch wer möchte von solchen Ausnahmen her die kritische Arbeit der Medien in der Kirche und für sie beurteilen? 


\section{Gottesrede und Gebet im Zeitalter der Massenmedien}

Es gibt in unserer gegenwärtigen Situation, quer durch Kirche und Gesellschaft, ein eigentümliches Phänomen, nämlich eine gesteigerte Religionsfreudigkeit, die gepaart ist mit einer schwelenden Gottesmüdigkeit, eine Ausbreitung der Meditationskultur bei gleichzeitiger Verödung der Gebetskultur. Wenn es vor mehreren Jahren einmal hieß: Jesus, ja - Kirche, nein, so scheint heute die Devise zu lauten: Religion, ja - Gott, nein. Es wäre wohl nicht schwer, diese neue Religion ohne Transzendenz, diese Religion der psychologisch-ästhetischen Seelenverzauberung als eine Art Kompensationsphänomen in unserer Massenmedienwelt darzustellen. Doch darum geht es mir nicht. Ich frage mich vielmehr: Was ist mit der Gottesrede, was mit den Gebeten geschehen, daß sie heute selbst im kirchlichen Raum so sehr ins Abseits gerieten? Woher die Atrophie der Gebete, die auch für viele Christen nicht einmal mehr Privatsache sind? Wie muß unsere Gottesrede gestaltet sein, wie unsere Gebete, wenn sie den Erfahrungen der Massenmedienwelt standhalten wollen?

Die Massenmedien präsentieren ein dramatisches Universum menschlicher Schicksale, sie liefern Schicksalsbilder, an denen sich die Rede zu Gott und über Gott bewähren muß. Von „meinem“ Gott kann da nur die Rede sein im Antlitz der anderen, der fremden anderen, aller anderen - eben im Blick darauf, daß Gott "mein“ Gott nur sein kann, wenn ich ihn auch als den Gott aller anderen anbeten kann, wie sie mir in diesem Schicksalsuniversum tagtäglich begegnen: also als den Gott der Fliehenden und Stürzenden, der Verhungernden und Verbrennenden ... Denn sind sie nicht alle in einem schlichten schöpfungstheologischen Sinn "Kinder Gottes“, Kinder des in meinen Gebeten gesuchten Vaters? Um das Gemeinte zu verdeutlichen, erinnere ich Sie an eine jüngste Fernsehsequenz: Bilder von den Stürmen in Bangladesh, in denen Hunderttausende anonym und antlitzlos hinweggefegt wurden; unmittelbar anschließend Bilder aus Fatima, in denen Papst Johannes Paul II. dem Himmel für seine Errettung aus dem früheren Attentat dankt. Hätte man in diesem Dank nicht auch die Klage hören müssen, die Klage über die untergehenden anderen? Das Massenmedium stellt ein unsere Gebete irritierendes Universum her. Dieses Schicksalsuniversum zwingt unsere christlichen Gebete zu einer besonderen Theodizeempfindlichkeit. Es treibt eigentlich jeden frommen Narzißmus aus. Es macht die Leiden und Katastrophen der anderen zum Stoff der eigenen Anbetung, einer Anbetung, in der das Lob nicht ohne Klage sein kann, der Dank nicht ohne Trauer und der Gesang nicht ohne Geschrei.

So aber hören sich die Gebete an in den Urschriften des Glaubens, so werden die Gebete von morgen sein oder sie werden nicht mehr sein. Für kleingläubig und verzagt wird diese Gebetssprache nur der halten können, der seine eigene Verblüffungsfestigkeit gegenüber den Katastrophen und Untergängen der anderen für verdienstvolle Glaubensfestigkeit hält. Das freilich wäre trügerisch. Die christliche Gotteserfahrung ist eingeschworen auf die Wahrnehmung des Schicksals der anderen. Darum auch ist die christliche Mystik in ihrem Kern keine Mystik der geschlossenen, sondern der schmerzlich geöffneten Augen. Sie mutet ein besonderes Exerzitium des Sehens zu, eine Überwindung unserer eingeborenen Sehschwierigkeiten und unserer kreatürlichen Narzißmen. Wer "Gott" sagt, nimmt die Verletzung der eigenen Gewißheiten durch das Unglück der anderen in Kauf. Das, so meine ich, sollten alle in Betracht ziehen, 
die mitten im Gewoge und unter dem anonymen Druck der Schicksalsbilder des Fernsehens das Wort „Gott" wagen. Dann vielleicht bricht sich, wenigstens für Augenblicke, an diesem ohnmächtigen Wort auch die Übermacht der Bilderwelt des Mediums.

\section{Ein strikter Kommunikationsvorbehalt: keine Eucharistie im Fernsehen!}

„Zum Wort hat auch der Ungläubige Zutritt, zum Sakrament nicht.“ Dieser Satz stammt von Dietrich Bonhoeffer (1937). Mit ihm möchte ich abschließend eine Grenze der massenmedialen kirchlichen Kommunikation benennen, eine Grenze, die inzwischen freilich längst überschritten wurde, einen Kommunikationsvorbehalt, der längst preisgegeben scheint. Ich bin, schlicht gesagt, der Meinung, daß das sakramentale Zentrum der Liturgie, daß die Feier der Eucharistie nicht ins Fernsehen gehört.

In den 50er Jahren gab es zeitweilig eine heftige Diskussion über die Frage, ob die Messe im Fernsehen übertragen werden dürfe, ob also die Fernsehkamera das sehen lassen und jedermann darbieten dürfe, was der gläubige Christ, der das Mysterium der Kirche leibhaftig mitfeiert, sehen darf und sieht. Diese Diskussion ist längst verstummt. Die Frage scheint inzwischen eindeutig entschieden. Schon 1971 resümiert unsere Pastoralinstruktion (Nr. 151): „Zum religiösen Programm (des Fernsehens) gehört sicherlich auch die Übertragung der Heiligen Messe ...". Sicherlich? Karl Rahner gehörte zu denen, die sich in diese frühe Diskussion der 50er Jahre eingeschaltet hatten. Nach ihm ist die ausführliche und volle Feier der Eucharistie ein bevorzugter Gegenstand jener metaphysischen Scham, die es verbietet, dieses Ereignis einer beliebig gestreuten und unbeteiligten Öffentlichkeit zugänglich zu machen. Welche Verletzungen dieser metaphysischen Scham durch Religion in unserer Öffentlichkeit möglich sind, kann man heutzutage nicht zuletzt an den Orgien metaphysischer Schamlosigkeit erkennen, wie sie die sog. 'electronic religion' vorführt. Ist die modische Esoterik, die Flucht in Okkultismus und Geheimbündelei, wie sie heute in unseren massenmedialen Gesellschaften allenthalben zu beobachten ist, nicht auch als anthropologischer Reflex zu deuten auf einen Veröffentlichungswahn, der alle metaphysischen Schamgrenzen längst abgesenkt, ja zum Verschwinden gebracht hat?

Wäre es also nicht an der Zeit, mit Dietrich Bonhoeffer und Karl Rahner an eine alte kirchliche Tradition zu erinnern, an die sog. Arkandisziplin? Bei ihr handelt es sich um eine Art Geheimhaltungspflicht bzw. Veröffentlichungsverbot, das sich in der frühen Christenheit vor allem auf die Feier der Eucharistie in der damaligen heidnischen Umwelt bezog. Es verschwand dann auch mehr oder minder, so ab dem 5. Jahrhundert, als es gewissermaßen keine Heiden, keine Ungläubigen mehr gab. Wäre aber heute, da im Fortgang von Aufklärung und Säkularisierung die gesellschaftliche und die kirchliche Öffentlichkeit längst wieder auseinandergetreten sind, die in dieser Arkandisziplin enthaltene Frage nicht erneut und neu zu stellen? Dietrich Bonhoeffer und Karl Rahner und wohl auch die neue politische Theologie, die sich ihnen hierin anschließt, bürgen $\mathrm{m}$. E. dafür, daß es sich bei dieser Erinnerung an die Arkandisziplin nicht um die Empfehlung einer neuen Ghettomentalität handelt, nicht um den Rückzug in ein 
neues kirchliches Privatissimum, nicht um den Verrat an der Weltsendung und am Öffentlichkeitsauftrag der Kirche. Wer hätte sich schon radikaler der Weltlichkeit unserer heutigen Welt ausgesetzt? Wer schon hätte entschiedener einen Glauben vorgetragen, hinter dem sich kein weltliches Privileg mehr verschanzt, als diese Theologen? Ist es vielleicht gerade dieses leidenschaftliche weltliche Engagement, das sie - und bislang nahezu ausschließlich sie - auf der Rettung des Arkanum, auf diesem Veröffentlichungsvorbehalt bestehen läßt? In den letzten Notizen Bonhoeffers aus dem Jahre 1944 steht die Frage: „Wie sprechen ... wir 'weltlich' von 'Gott', wie sind wir 'religionslos-weltliche' Christen, wie sind wir ek-klesia, Herausgerufene, ohne uns religiös als Bevorzugte zu verstehen, sondern als ganz zur Welt Gehörige? ... Bekommt hier die Arkandisziplin ... neue Wichtigkeit?" Bonhoeffer schließt dann die Forderung an: „... es muß eine Arkandisziplin wiederhergestellt werden, durch die die Geheimnisse des christlichen Glaubens vor Profanierung behütet werden." Diese Arkandisziplin bedeutet ihm offensichtlich nicht Abschied und Absonderung von der Welt, sondern Konzentration auf das Geheimnis der Welt. "Nicht die platte und banale Diesseitigkeit meine ich", schreibt Bonhoeffer vor seiner Hinrichtung, „sondern die tiefe Diesseitigkeit, ... in der die Erkenntnis des Todes und der Auferstehung immer gegenwärtig ist." Augenscheinlich soll durch diese Arkandisziplin die eucharistische Erinnerung des Todes und der Auferstehung geschützt werden, damit sie als gefährliche Erinnerung in der Öffentlichkeit einer Welt wirken kann, in der die Menschen immer weniger aus ihren Erinnerungen, sondern immer mehr aus ihren Reproduktionen und als ihr eigenes Experiment leben.

Was denn, um Gottes willen, hat unsere Kirche bewogen, jeglichen Kommunikationsvorbehalt angesichts der Massenmedienwelt preiszugeben? Selbst die profane Gesellschaft kennt einen Datenschutz; die Kirche, so sieht es aus, kennt nicht einmal mehr einen Geheimnisschutz. Wie konnte es dazu kommen? Wie dazu, daß die Kirche nicht grundsätzlich unterscheidet zwischen einer partizipatorischen Öffentlichkeit bei der Feier des Geheimnisses selbst und deren reproduktiver Veröffentlichung in einer strikt egalitären Massenkultur, in deren Selbstdefinition "Geheimnis“ nicht vorkommt - ?

Hängt die offizielle Kirche hier einer zu naiven Medientheorie an? Vermutlich schon. Selbst wenn man sich der heute insbesondere von Neil Postman verführerisch illustrierten Auffassung Marshall McLuhans, wonach ausschließlich das Medium die Inhalte determiniert, nicht einfach anschließen mag, und auch wenn man Jean Baudrillards Simulationstheorie mehr für einen brillianten Gag als für ein beweisfähiges Argument hält, wird man doch auch die extreme Gegenposition, wonach allein die Inhalte und die Intention der Gestalter das an sich neutrale Medium prägen, sehr skeptisch betrachten müssen. Allzu leicht unterstützt man sonst das peinliche Gegenteil von guten Sendungen, nämlich gut gemeinte Sendungen. Das Medium selbst erfordert größte Aufmerksamkeit. Das wrßte schon Walter Benjamins Kunstwerkthese. Schon von ihr könnte man lernen, daß durch die Reproduktionsmedien zwei für die kultische Erfahrung konstitutive Elemente verlorengehen: einmal die Authentizität, weil das Medium die Differenz zwischen Original und Kopie aufhebt, und dann die Tradition, weil das reproduzierende Medium seine Inhalte den zeitlichen und räumlichen Zusammenhängen entnimmt; Reproduktion tritt an die Stelle der Tradition, d. h. sie besetzt diese Stelle. Authentizität und Tradition können nur, wenn über- 
haupt, durch leibhaftige Partizipation gerettet werden. Sie müssen es mir erlassen, das eben Gesagte über diesen Hinweis hinaus noch auszuführen.

Ich will nur nochmals kurz auf die Frage zurückkommen, was denn die Kirche mit ihrer zentralen Kultfeier in die massenmediale Öffentlichkeit treibt. Will sie mit dem Zentrum ihrer Liturgie „werben“ oder „evangelisieren“? Hat sie Angst, ansonsten nicht genügend präsent zu sein? Müßte sie nicht vielmehr Angst haben vor dem geradezu tödlichen Abnützungseffekt der Fernsehgegenwart? Und weiß sie nicht, daß, je unentrinnbarer, je quasi omnipräsenter die Medien für uns werden, umso aufregender und attraktiver eines Tages das sein wird, was in ihnen nicht zu besichtigen ist? Gewiß, die Kranken, die Alten und Einsamen: für sie, sagt man, sei die Eucharistie im Fernsehen da. Doch einmal davon abgesehen, daß man Regeln nicht von ihren Ausnahmen her ableiten sollte, abgesehen davon drängt sich die Frage auf, ob hier die Massenkommunikation nicht zu schnell ersetzen soll, was eigentlich an personaler Kommunikation, also an persönlichem Kranken- und Altenbesuch pastoral gefordert wäre?

Ich werde den Verdacht nicht los, daß die Kirche, die sich ansonsten nur sehr zögerlich modernisiert, hier in die Falle eines gefährlichen Modernismus getappt ist. Daraus würde ich sie - mit Ihrer Hilfe - gern befreien. Ich glaube, das käme einer unbefangeneren produktiven Medienarbeit in allen anderen Kommunikationsbereichen nur zugute.

\section{SUMMARY}

The Second Vatican Council initiated perspectives of a cultural polycentric "Worldchurch" because at our times the Church is on its way to a real worldwide community. At the same time, however, the Church gets into the position of a minority, of a global diaspora. Whether the Church succeeds on becoming a cultural polycentral "Worldchurch" depends definitely from the recognition of other cultures in their own originality; which means developping a pretentious communicative culture beyond every kind of assimilation or domination. The Church must warn of the danger of a "secondary colonisation" of people in the Third World by the European technology of information and communication which makes western values and meanings more and more dominating the universal world.

Instead of symptoms of a new sectirian and Ghetto mentality, new ways of life within the Church are required as well as a sober, unsentimental pastoral of diaspora. In this connection the Church has to examine critically also the new delight in the religious feelings of the present cultures of meditation. These cultures go along with a sneaking loss of the Church-teachings of God's words and of the Church-culture of praying which always includes the sufferings and tragedies of others. From this points of view, Metz makes a strict reservation in communication: No transmission of Eucharistic Mass in Television! Against the usual transmitting of Masses, he cites (the German Protestant Theologian) D. Bonhoeffer: ${ }_{\text {To the }}$ word (of God) also infidel people have access but not to the sacrament". Following the (German Theologian) Rahner, Metz points out that the celebration of the Eucharisty belongs into the realm of a ${ }_{n}$ metaphysical shame " which prohibits a presentation to any dispersed and unconcerned public. At the end, Metz puts the question whether the Church in agreeing to transmission of Masses did not fall into the trap of a dangerous modernism.

\section{RESUMÉE}

Le concile Vatican II. conçut dans ses grandes lignes initiales la perspective d'une Eglise universelle polycentrique au niveau culturel, car elle est pour la premiere fois en 
voie de devenir une veritable Eglise universelle. Mais en même temps, elle se retrouve partout en situation de minorité, formant une diaspora mondiale. Mais la réussite de l'Eglise en tant qu'Eglise universelle polycentrique au niveau culturel dépend essentiellement du fait de savoir si elle est à même de développer en son sein une culture qui reconnaisse les autres, dans leur façon d'être différente, donc de développer une culture communicative exigeante, surmontant toute forme de culture d'assimilation et de domination. Ce faisant, l'Eglise doit mettre en garde contre le danger d'une "colonisation secondaire" des pays du Tiers-Monde par la technologie européenne en matière d'information et de communication, car c'est à travers cette dernière que les valeurs et le idées protant l'emreinte de l'Occident prennent peu à peu la mainmise universelle sur le monde.

Contre les symptômes d'une nouvelle mentalité de secte et de ghetto au sein de l'Eglise, il importe de'elaborer des nouvelles formes de vie religieuse ainsi qu'une pastorale de la diaspora objective et absente de sentimentalité. En même temps, l'Eglise doit aussi examiner de façon critique le nouvel engouement pour la religion et la culture de méditation actuelle, car il va de pair avec le fait que, dans l'Eglise, la parole de Dieu et la tradition de la prière se perdent lentement, dans lesquelles les souffiances et les catastrophes éprouvées par les autres donnent toujours, elles aussi, matière à la prière personnelle. Dans ce contexte, Metz plaide pour une stricte réserve de communication: pas de fête de l'eucharistie à la télévision! A l'encontre de la pratique courante de retransmettre l'office divin à la télévision, il rappelle une parole de D. Bonhoeffer: „Si le mécréant a lui aussi accès à la parole, il ne l'a pas au sacrement“. Dans le même esprit que Rahner, l'auteur fait remarquer l'office divin tombe dans le domaine d'une „pudeur métaphysique" interdisant de donner accès à cet événement à un public aussi disséminé et in différent qu'il soit. Pour finir, Metz demande si, avec les retransmissions de l'office divin à la télévision, l'Eglise n'est past tombée dans le piège d'un modernisme dangereux.

\section{RESUMEN}

El Concilio Vaticano II. concibió la perspectiva de una Iglesia universal culturalmente policéntrica, así por primera vez se encuentra en el camino de convertirse en una Iglesia realmente transformadora del mundo. Al mismo tiempo, en todas partes, está cayendo en situaciones de minoría, en una diáspora global. El que la Iglesia consiga constituirse en una Iglesia universal, culturalmente policéntrica depende determinantemente de que, dentro de ella, pueda desarrollarse una cultura de reconociemiento del oro como ser diferente, o sea, si puede desarrollar una cultura comunicativa seria que supere todo tipo de cultura de dominio y asimilación. Al mismo tiempo la Iglesia tiene que advertir del peligro de „una secundaria colonización“ de los países del Tercero Mundo a través de la tecnología de comunicación y información europea marcada por los valores y opiones occidentales que poco a poco asumen el dominio total del mundo.

Contra los síntomas de una mentalidad nueva de secta o gueto en la Iglesia, hay que desarrollar nuevas formas de vida eclesiales y una diáspora no sentimental y sobria. Por otro lado, la Iglesia debe examinar también criticamente el nuevo entusiasmo por la religión de la actual cultura de la meditación porque ésta va acompanada por la pérdida de la palabra de Dios y de la cultura de la oración convirtiéndose siempre los sufrimientos y las catástrofes de los demás en materia de la propia oración. En este contexto Metz aboga por una estricta reserva en la comunicatión: ¡Ninguna celebración eucarística por televisión! Contra la práctica de uso corriente de transmisiones de misas por televisión nos recuerda unas palabras de D. Bonhoeffer: „A la palabra también tiene acceso el no creyente, al sacramento, no". Enlazando con Rahner el autor manifiesta que la misa en el campo de una "vergüenza metafísica", que prohibe poner al alcance este acontecimiento a un público no participante, disperso y arbitrario. Finalmente Metz hace la pregunta de si la Iglesia con las transmisiones de las misas no ha sido cazada en la trampa de un peligroso modernismo. 